\title{
La réduction fait peur
}

\section{Anna Sax}

Lic. oec. publ., MHA, membre de la rédaction

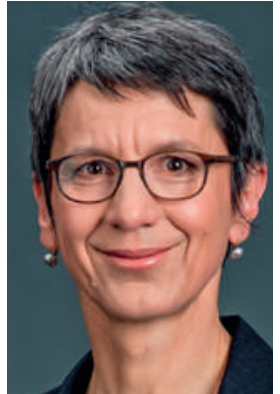

Quand on cherche de l'inefficacité et des incitations erronées dans les soins de santé, on a de bonnes chances d'en trouver: en médecine, les prestations inutiles s'enchaînent. On diagnostique, soigne et opère trop. Une petite décennie après les débats animés sur le rationnement dans le secteur de la santé, la nouvelle formule magique s'intitule à présent: «Moins, c'est mieux.» Le symposium* 2015 de l'ASSM se demandait entre autres comment la recherche sur les services de santé pourrait contribuer à résoudre le problème de la surabondance de soins.

Nikola Biller-Andorno, Professeure à l'Institut d'éthique biomédicale de l'Université de Zurich, a ouvert la discussion avec une déclaration sur l'appropriateness. Ses réflexions ont d'abord porté sur la signification de l'appropriate care, c.à-d. des soins appropriés, et elle a posé, comme définition de travail, le postulat du triangle d'objectifs santé publique, dépenses par personne et experience of care. Elle a souligné qu'il fallait garder ces trois objectifs à l'esprit pour que les soins de santé puissent être réalisés de manière adéquate. Les deux premiers sont faciles à comprendre. La recherche d'une traduction de l'expression experience of care

\section{Qui exactement définit ce qui est appropriate, c.-à-d. adéquat et opportun?}

montre par contre à quel point cette notion est difficile à transmettre. Le NHS, service national de santé britannique, fait ce constat plutôt lapidaire sur son site Internet: "Experience of care means many things to many people.» Sur la base des explications complémentaires du NHS, je conclus qu'il s'agit plus ou moins de la façon dont les gens s'en sortent avec le système de santé, des expériences qu'ils y font et de la manière dont cela les aide à retrouver ou à conserver la santé. La conférencière et son public se sont ensuite demandé: Qui exactement définit ce qui est approprié, c.-à-d. adéquat et opportun? Qui sont les parties prenantes qu'il convient de servir? Oui, bien sûr, ce sont les patients, comment avons-nous pu l'oublier? Oui, mais ne s'agit-il que d'eux ou aussi des personnes en bonne santé - les patients potentiels - autrement dit toute la population? Et d'où cette dernière tire-t-elle ses informations? On le sait, l'opinion publique peut être manipulée, BillerAndorno l'a rappelé en donnant l'exemple des programmes de dépistage ou de la médecine complémentaire. Elle a demandé des preuves et des études unbiased. Je me suis alors dit: et si les gens ne voulaient pas de soins de santé appropriés?

Alors pourquoi, me suis-je une fois de plus demandée, les choses ne bougent-elles pas davantage?

Steve Pearson, Professeur de bioéthique de Bethesda (Etats-Unis), a apporté de premiers éléments de réponse. Pour lui aussi, le waste ou gaspillage est partout. Il a expliqué pourquoi il est si difficile de renoncer au superflu en dépit de la raison: la réduction basée sur les faits aboutit au one-size fits all, à une taille unique, niant le besoin d'individualité des patients. Le Professeur Nicolas Rodondi de l'hôpital de l'Ile a vanté les mérites du projet Smarter Medicine de la SSMI, qui met en évidence des interventions souvent réalisées mais dont l'utilité ne peut être prouvée et auxquelles il convient donc de renoncer à l'avenir. Beaucoup sont des prestations auxquelles patientes et patients se sont habitués et qui renforcent leur confiance dans le médecin. Le Professeur Gianfranco Domenighetti, absent pour cause de maladie, a souligné par personne interposée l'impopularité en Suisse de la recherche sur les services de santé.

Le symposium réunissait beaucoup de gens qui ont leur mot à dire et pourraient changer les choses dans le système de santé s'ils le voulaient vraiment: médecins-chefs, professeures, chercheurs, représentantes des autorités cantonales et nationales, des assurances, des hôpitaux et de l'industrie. Alors pourquoi, me suis-je une fois de plus demandée, les choses ne bougent-elles pas davantage? Finalement, tout à la fin de la table ronde, alors que près d'un quart des participants était déjà parti, une représentante des patients a obtenu la parole en la personne de la conseillère nationale Bea Heim. Ses déclarations ont en quelque sorte apporté la réponse suprême à mes questions: "Less is more - c'est précisément de cela que les patientes et patients ont peur.» 